\title{
Metastatic colorectal carcinoma to one ovary with vanishing primary: A case report in an 18-year- old patient
}

\author{
Jordy Mehawej ${ }^{1}$, Nicole El Helou ${ }^{2}$, Tanja Pejovic ${ }^{3}$ and Paulette Mhawech-Fauceglia ${ }^{4 *}$ \\ ${ }^{1}$ Department of Medicine at University of Massachusetts Medical School, Worcester, MA, USA \\ ${ }^{2}$ Department of Obstetrics and Gynecology at Washington University, St. Louis, MO, USA \\ ${ }^{3}$ Division of Gynecologic Oncology at Oregon Health \& Science University, Portland, Oregon, USA \\ ${ }^{4}$ Department of Anatomic Pathology at Aurora Diagnostics/Sonic Healthcare, Austin, TX, USA
}

\begin{abstract}
An 18-year-old young woman with chief complaint of left rib pain and increasing girth. An abdominal CT-scan showed a $34 \mathrm{x} 26 \mathrm{~cm}$ complex right pelvic mass. Due to the young patient's age, a fertility preservation surgery was recommended, and unilateral salpingo-oophorectomy was planned but then proceeded to tumor staging. Microscopically, the mass was composed of back to back glands, lined by hyperchromatic nuclei and prominent nucleoli, with ample mucin cytoplasm. Immunohistochemistry showed tumor positive for pan cytokeratin (AE1/3), CK20, CDX2 and negative for CK7, CD30, calretinin, glypican, PAX8, ER/PR, $\alpha$ fetoprotein (AFP), $\alpha$-inhibin, Placental phosphatase protein (PLAP), OCT4, EMA and p53. There was no loss of mismatch repair proteins in tumor cells. The final diagnosis was metastatic adenocarcinoma from colorectal primary. Subsequently, the search for the primary carcinoma returned negative. The patient just finished 5 cycles of adjuvant FOLFOX therapy (5-fluorouracil and Oxaliplatin) with no side effects.
\end{abstract}

\section{Case report}

A pleasant 18-year-old, G0, who was brought into the emergency room due to a left rib pain that started 2 weeks prior and worsened over time. The patient noticed an abdominal swelling over the last $2-3$ months which she attributed to a weight gain. She denied loss of appetite. Her past medical history is negative. At physical examination, the abdomen was distended with a notable non tender mass extending to her xiphoid. A CTscan showed a $34 \times 26 \mathrm{~cm}$ complex partly cystic and partly solid right pelvic mass that most likely compressing the ureter and leading to slight hydronephrosis. No enlarged lymph nodes and no ascites were present. Her laboratory data were within normal limit. Given the patient age, a fertility preservation surgery was recommended but the surgeon has proceeded to tumor staging with lymph node dissection, omentectomy and peritoneal biopsies. The specimen consisted of an ovarian mass weighing 1443 grams and measuring $32 \times 26 \times 12 \mathrm{~cm}$. The outer surface was smooth and glistening. The cut surface was partly cystic and partly solid with areas of hemorrhage and necrosis. The attached fallopian tube was unremarkable. Microscopically, the ovarian mass exhibited back to back glands, lined by hyperchromatic nuclei and prominent nucleoli, with ample mucin cytoplasm. In places, the glands were dilated and filled with eosinophilic necrotic debris, so called "dirty necrosis" (Figure 1). No lymphatic invasion was seen. Immunohistochemistry (IHC) showed tumor cells positive for pan cytokeratin (AE1/3), CK20, CDX2 and negative for CK7, CD30, calretinin, glypican, PAX8, ER/PR, afetoprotein (AFP), a-inhibin, Placental phosphatase protein (PLAP), OCT4, EMA and p53. IHC showed no loss of the mismatch repair proteins MLH1, MSH2, MSH6 and PMS2. The fallopian tube and the appendix were submitted entirely and they were unremarkable. The pelvic washing, omentum, right and left pelvic and periaortic lymph nodes as well as biopsies from the peritoneum, bladder, cul de sac, pelvis, and left gutter, and appendix

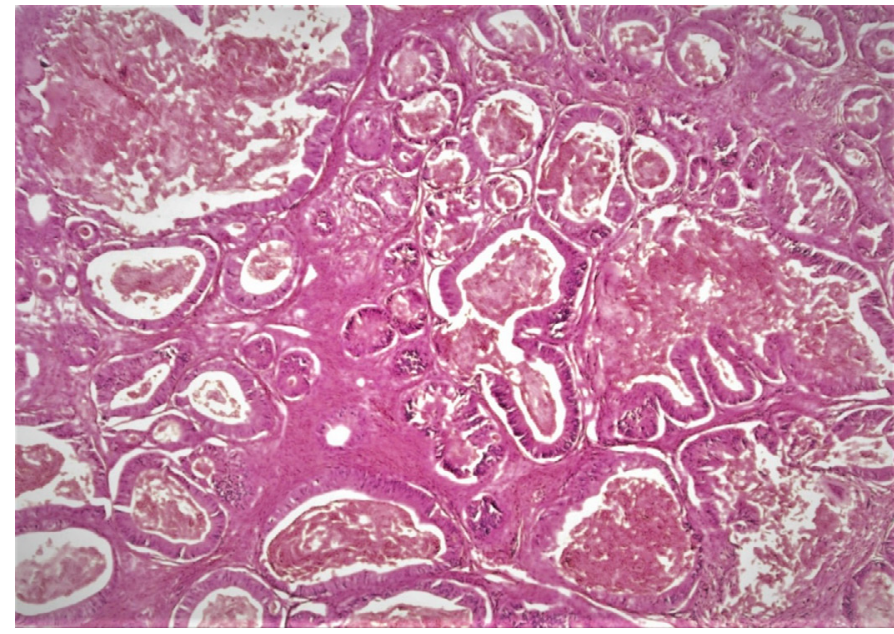

Figure 1. Hematoxylin-eosin section of the ovarian mass showed tumor composed of glands of different size. The glands are lined by hyperchromatic nuclei with big nuceoli and ample cytoplasm. The glands lumen are filled with necrotic eosinophilic debris "dirty necrosis" (x20)

were all negative for tumor. Based on the morphology and the IHC profile, the final diagnosis was metastatic adenocarcinoma, most likely from colorectal primary. The case was sent to two different institutes for

${ }^{\star}$ Correspondence to: Paulette Mhawech-Fauceglia, Gynecologic Pathology consultant, $12221 \mathrm{~N}$ Mopac Expressway, Austin, Texas 78758, USA, E-mail: pfauceglia@auroradx.com

Key words: young age, metastatic carcinoma, ovary, regression of primary, next generation sequencing

Received: March 31, 2020; Accepted: April 10, 2020; Published: April 15, 2020 
second opinion and an agreement with the above diagnosis was reached. Searching for primary tumor, an esophagogastroduodenoscopy (EGD) and colonoscopy were done and they were negative. Three days after her endoscopy the patient experienced chest and abdominal pain. Chest CT-scan showed a left airspace disease consistent with pneumonia. Abdominal CTscan showed a free intraperitoneal gas, necessitating post-exploratory laparotomy and rigid rectoscopy and they showed normal appearing colon, small intestine and stomach with absence of spillage, or leak. A nasogastric tube was placed and she was started on antibiotics. After short hospitalization, the patient recovered well and she was discharged home. Paraffin-embedded tumor tissue block was sent for next generation DNA-sequencing (NGS) and the results showed low burden of tumor mutation, no genes mutation, absence of microsatellite instability, and wild types KRAS, NRAS, HRAS and $B R A F$. The patient has just finished her fifth cycle of adjuvant FOLFOX therapy (5-fluorouracil and Oxaliplatin) which was well tolerated with no side effects. Patient is planning to come back to complete her remaining treatment.

\section{Discussion}

We reported a very unique case of metastatic adenocarcinoma to one ovary in a very young woman from vanishing/regressing colorectal carcinoma. NGS revealed tumor with low mutation burden and no gene mutations.

The ovary is a common site for metastasis as $3-15 \%$ of ovarian tumors are of metastatic origin with the most common primaries are of colorectal, gastric, pancreaticobiliary tract, and breast origin. The diagnosis relies on numerous pillars such as clinical data, gross finding, microscopic evaluation and immunohistochemistry (IHC) profile. Approximately 3-7 \% of colon cancer patients have metastatic ovarian cancer at the time of initial presentation. In comparison to primary ovarian carcinoma, the gross findings in CRC, include bilaterality in more than $60 \%$ of cases, small size $(<10-12 \mathrm{~cm})$, nodular growth pattern, and tumor on the surface of the ovary or superficial cortex of ovary [1]. In contrast, the tumor in our case was unilateral, very big of $34 \mathrm{~cm}$, confluent and with no surface involvements. In addition, CRC occurs in different age group between 50-90 years old. At this stage, all indications appeared favoring primary ovarian cancer. Our differential diagnosis was most likely germ cell tumors, sex cord stromal tumors, surface ovarian tumors and least likely metastatic carcinomas. Microscopically, the ovarian tumor was composed of glands of moderate size ranging from small to large dilated glands and the most characteristic finding is the eosinophilic necrotic material with karyorrhetic debris as known as "dirty necrosis" supporting the diagnosis of metastatic CRC [2]. To confirm our microscopic hypothesis, a vast IHC panel excluded germ cell tumors (CD30-, CK7-, a-fetoprotein-, PLAP -, OCT 4-) and sex cord stromal tumors (calretinin- and a-inhibin-). Furthermore, IHC profile indicated that the tumor was not of primary Mullerian origin as CK7-, PAX8- but in the contrary it is from the GI tract and more specifically CRC (CK20+, CDX2+). IHC panel to differentiate primary ovarian adenocarcinoma (endometrioid/mucinous) from metastatic CRC has been widely explored and an IHC panel such as CK7+/ CK20, CDX2-, ER+/PR+ and PAX8+; supports a CRC primary with more than $99 \%$ accuracy which what was seen in our case [3-6] (Figure 2).

All exploration such as EGD, colonoscopy, rectoscopy and exploratory laparoscopy after surgery failed to find a primary tumor. Vanishing tumor or tumor regression was reported in prostate where cancer was seen in needle biopsies to totally disappear on radical prostatectomy specimen [7]. A review of literature showed very few
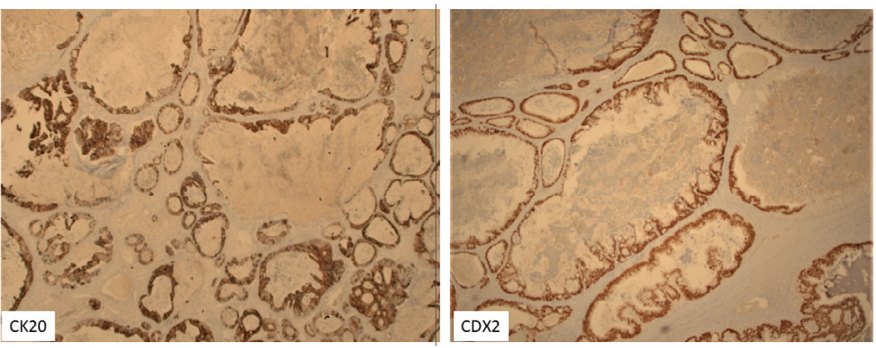

Figure 2. Immunostain showed tumor cells to be CK20+, CDX2+

cases of spontaneous regression of colorectal carcinoma without being ever treated [8]. We believe that our patient fall in this very rare category. Other uniqueness of our patient is her very young age of only 18 years old where CRC are very rare occurrence with an incidence of 1:1million [9]. Even more, CRC metastatic to one ovary in this age group is almost non-existent and no cases have been reported till today. CRC in adolescent is often seen in a setting of hereditary cancer syndromes where the incidence is very high of $60 \%$ in lynch syndrome and $90 \%$ in familial adenomatous polyposis in comparison to $10 \%$ in general population. However, in our patient there is no family history of such a cancer and the tumor was microsatellite stable. Recent review of CRC in children and adolescents were conducted indicating that they have similar natural history but they are often seen in advanced stage disease at diagnosis, higher occurrences of aggressive tumors and poorer outcome. Due to their rarity, they are often overlooked leading to the delay in diagnosis and treatment. The FOLFOXregimen is the treatment of choice. The prognosis is often poor due to stage IV disease. Next generation DNA sequencing has been used to guide oncologists for targeted therapy. In our case NGS howed very low mutation burden and absence of genes mutations including $k R A S$ and NRAS. Approximately $30-50 \%$ of CRC are known to have mutated KRAS gene which means up to $50 \%$ of patients might respond to anti-epidermal growth factor receptor (EGFR)antibody therapy [10]. Due to the patient tumor wild type of KRAS and BRAF, it suggests that the tumor is sensitive to antiEGFR antibodies that could be considered in the future.

In summary, this is unique caseof metastatic adenocarcinoma to the ovary form the colorectal primary, withunilateral involvement of the ovary, the very young patient age and finally the vanishing/regressing primary and the low genetic mutation burden and the absence of genes mutation.

\section{Author contribution}

Data Curation: Paulette Mhawech-Fauceglia

Investigation: Paulette Mhawech-Fauceglia

Supervision: Paulette Mhawech-fauceglia

Writing- original draft: Jordy Mehawej, Nicole El-Helou

Writing-review and Editing: Tanja Pejovic, Jordy Mehawej, Nicole El-Helou

\section{Conflict of interest}

The authors have no conflict of interest

\section{References}

1. Lee SJ, Bae JH, Lee AW, Tong SY, Park YG, et al. (2009) Clinical characteristics of metastatic tumors to the ovaries. J Korean Med Sci 24: 114-119. [Crossref]

2. Female genital tract tumors; 2014 WHO. 
3. Kriplani D, Patel MM (2013) Immunohistohemistry: A diagnostic aid in differentiating primary epithelial ovarian tumors and tumors metastatic to the ovary. South Asian J Cancer 2: 254-258. [Crossref]

4. Park SY, Kim BH, Kim JH, Lee S, Kang GH (2007) Panels of immunohistochemical markers help determine primary sites of metastatic adenocarcinoma. Arch Pathol Lab Med 131: 1561-1567.

5. Grosman GM, Bernheim J, Halperin M, Brazowsky E, Meir A (2005) Expression of the intestinal maerker cdx2 in secondary adenocarcinoma of the colorectum. Arch Pathol Lab Med 129: 920-923.

6. Cathro HP, Stoler MH (2002) Expression of cytokeratins 7 and 20 in ovarian neoplasia. Am J Clin Pathol 117: 944-951.
7. Bostwick DG, Bostwick KC (2004) Vanishing' prostate cancer in radical prostatectomy specimens: incidence and long-term follow-up in 38 cases. BJU Int 94: 57-58. [Crossref]

8. Shimizu H, Kochi M, Kaiga T, Mihara Y, Fuji M, et al. (2010) A case of spontaneous regression of advanced colon cancer. Anticancer Res 30: 2351-2354. [Crossref]

9. Mirchandani D, Kulpa J, Khawar N, Kochia I, Narula P, et al. (2016) Metastatic colon cancer in an 19-year old without predisposing factors. Case Rep Pediatr 2016: 7820367. [Crossref]

10. Di Nicolantonio F, Martini M, Molinari F, Sartore-Bianchi A, Arena S, et al. Wild-type $\mathrm{BRAF}$ is required for response to panitumumab or cetuximab in metastatic colorecta cancer. J Clin Oncol 26: 5705-5712. [Crossref]

Copyright: (C2020 Mehawej J. This is an open-access article distributed under the terms of the Creative Commons Attribution License, which permits unrestricted use, distribution, and reproduction in any medium, provided the original author and source are credited. 